\title{
Establishment of F1 hybrid mortality in real time
}

\author{
Ashley Saulsberry ${ }^{1,2}$, Marisa Pinchas ${ }^{1,3}$, Aaron Noll ${ }^{1}$, Jeremy A. Lynch ${ }^{4}$, Seth R. Bordenstein ${ }^{1,5^{*}}$ \\ and Robert M. Brucker ${ }^{1,6^{*}}$
}

\begin{abstract}
Background: Measuring the evolutionary rate of reproductive isolation is essential to understanding how new species form. Tempo calculations typically rely on fossil records, geological events, and molecular evolution analyses. The speed at which genetically-based hybrid mortality arises, or the "incompatibility clock", is estimated to be millions of years in various diploid organisms and is poorly understood in general. Owing to these extended timeframes, seldom do biologists observe the evolution of hybrid mortality in real time.

Results: Here we report the very recent spread and fixation of complete asymmetric $F_{1}$ hybrid mortality within eight years of laboratory maintenance in the insect model Nasonia. The asymmetric interspecific hybrid mortality evolved in an isogenic stock line of $\mathrm{N}$. Iongicornis and occurs in crosses to $\mathrm{N}$. vitripennis males. The resulting diploid hybrids exhibit complete failure in dorsal closure during embryogenesis.

Conclusion: These results comprise a unique case whereby a strong asymmetrical isolation barrier evolved in real time. The spread of this reproductive isolation barrier notably occurred in a small laboratory stock subject to recurrent bottlenecks.
\end{abstract}

Keywords: Nasonia, Hybrid incompatibility, Development, Reproductive isolation, Speciation

\section{Background}

Given the importance of determining the patterns that affect the tempo of speciation, renewed emphasis has been placed on understanding how fast reproductive isolation barriers evolve during the speciation process. In the laboratory, complete $F_{1}$ hybrid mortality has never been documented to evolve de novo, while there are a few cases of incomplete premating isolation [1-4] and a case of hybrid reproduction defects in experimentally evolved yeast [5]. In mammals and birds, the evolution of strong hybrid mortality takes, on average, four million and 21 million years [6-8], respectively. In frogs, estimates suggest the minimum age for total hybrid inviability to become fixed is 1.5 million years $[9,10]$. However, incomplete reproductive isolation and/or segregating variation for hybrid incompatibles can occur within various species, such as Arabidopsis [11] and Tribolium [12]. Intraspecific variation in hybrid incompatibilities indicates that there is segregating variation for hybrid incompatibility

\footnotetext{
* Correspondence: s.bordenstein@vanderbilt.edu; bruckerm@gmail.com 1 Department of Biological Sciences, Vanderbilt University, Nashville, TN 37235, USA

Full list of author information is available at the end of the article
}

alleles, but it does not inform when the incompatibility factors arose or spread per se. Taken together, the estimated timespans for severe hybrid mortality to fix in natural populations suggests that its emergence is often slow and/or restricted to large populations.

The genus Nasonia includes four closely related species of parasitic wasps that diverged between 0.3 to 1.0 million years ago [13]. These species include $N$. vitripennis, $N$. longicornis, $N$. giraulti, and $N$. oneida. The latter three species evolved sympatrically within the geographic range of $N$. vitripennis. The sister species, $N$. giraulti and $N$. oneida, occur sympatrically and share a distribution in the North Eastern temperate zone of North America, while N. longicornis lives in allopatry from $N$. giraulti and $N$. oneida on the western side of the continent. All four species are commonly used in laboratory studies of interspecific differences in their genetics, phenotypes, and microbial symbionts (reviewed in [14]).

Multiple Wolbachia infections exist within Nasonia and cause cytoplasmic incompatibility between the species $[15,16]$. When reared under the same conditions in and before 2000 and cured of their Wolbachia infections, 
$N$. longicornis and $N$. vitripennis produced similar numbers of viable $F_{1}$ hybrid offspring (90-100\%) in comparison to parental controls [16] (Fig. 1a). Indeed, all intraspecific crosses in the Nasonia genus readily produce $F_{1}$ hybrids in the absence of Wolbachia [15-17]. However, hybrid breakdown is commonly observed in the haploid $\mathrm{F}_{2}$ hybrid males wherein cytonuclear incompatibilities and host-microbiota interactions cause hybrid larval mortality [18-21].

Recently, while attempting to perform experiments on $F_{2}$ hybrid breakdown between laboratory strains of Nasonia longicornis and Nasonia vitripennis, we discovered a novel $F_{1}$ hybrid lethality between these species (Fig. 1b). It is asymmetric, complete, and occurs in the cross between $N$. vitripennis males and $N$. longicornis females. This new and unexpected $F_{1}$ hybrid malady affords an opportunity to time the evolution of postzygotic isolation in the lab and to dissect its genetic basis. Here we describe several genetic and developmental analyses that led to timing the spread of this severe $F_{1}$ hybrid embryonic lethality within eight years of laboratory maintenance.

\section{Methods}

\section{Strains}

All Nasonia wasps were reared in $25{ }^{\circ} \mathrm{C}$ incubators with constant light on Sarchophaga bullata fly pupae ('hosts') raised in the lab. All fly hosts were always checked for color and firmness prior to providing them to adult female Nasonia to ensure the quality was sufficient for parasitism. This included a dark amber to brown puparium with yellow, firm to the touch, fly pupae inside (approximately 13 days old post egg laying). Two strains of $N$. longicornis were used in this study: IV7R3-1b (R3) and NAS_NLUT230A (UT). Strain IV7R3-1b is derived from strain IV7, a Wolbachia infected strain that was collected in Utah and antibiotically cured of Wobachia in 2000 [16]. NAS_NLUT230A was collected from a natural population in Utah between 1989 and 1991, cured of Wolbachia by antibiotic treatment, and maintained in the laboratory according to methods described previously [22]. Two strains of $N$. vitripennis were used in this study: 13.2 and 12.1T. Strain 13.2 was derived from the R511 line collected in New York and cured of Wolbachia through a period of prolonged diapause in 1996 [23]. The Wolbachia-uninfected 12.1T strain was derived after antibiotic treatment from strain 12.1 that harbors a Wolbachia infection [24] and originally derived from R511 [23]. The generation time for $N$. longicornis and $N$. vitripennis under these rearing conditions is approximately two weeks.

\section{Collecting and mating Nasonia}

All Nasonia stocks were matured into the yellow pupa stage at approximately twelve days of age and then

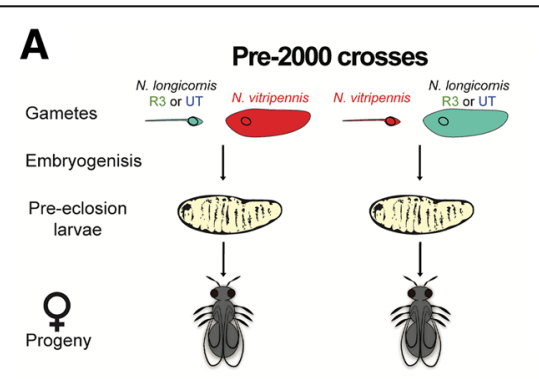

\section{B Post-2000 crosses}
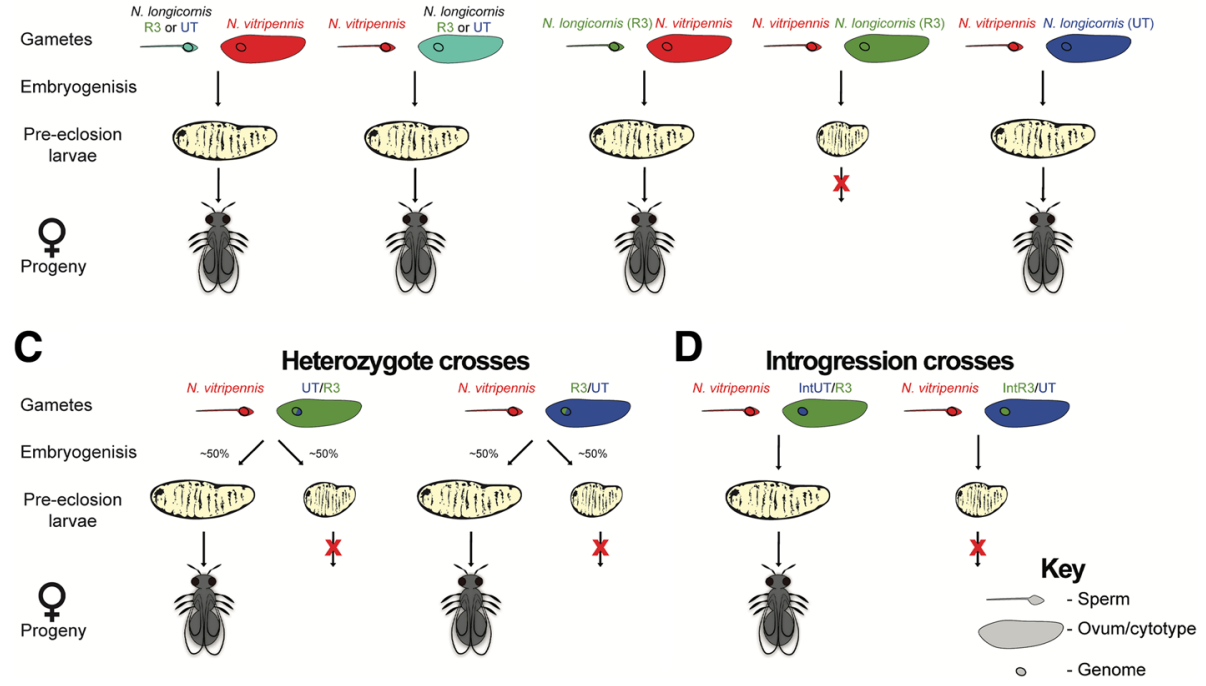

Fig. 1 Diagram of experimental crosses and their outcomes. a Interspecific crosses of Nasonia vitripennis and N. longicornis strains R3 and UT produce $F_{1}$ hybrids prior to 2008. b An asymmetric, postzygotic $F_{1}$ hybrid lethality arises between $N$. vitripennis strains and $N$. longicornis strain R3 but not with N. Iongicornis strain UT. c To test if this asymmetric hybrid lethality is due to a maternal genetic or cytoplasmic effect, heterozygous offspring of $N$. longicornis (UT/R3 and R3/UT) were crossed with N. vitripennis, resulting in approximately $50 \%$ survival of $F_{1}$ hybrid female offspring with both genotypes and cytotypes, indicating the hybrid incompatibility is not due to the R3 cytotype. d To further test the maternal genetic effect on hybrid mortality, N. longicornis strains UT and R3 were reciprocally backcrossed to each other, resulting in $\sim 99.8 \%$ genome replacement into the alternative line's cytotype. Females of these strains were then mated with $N$. vitripennis males, resulting in the same $F_{1}$ hybrid lethality observed when the R3 genome was in a UT cytotype (IntR3/UT) but not when the UT genome was in the R3 cytotype (IntUT/R3) 
separated according to sex to ensure virginity. After pupae eclosion, adult wasps were allowed to feed on small amounts of honey. For experimental mating crosses, one adult male and one virgin adult female Nasonia of the desired strains were each placed into a glass test-tube vial that was capped with a cotton plug. The pairs were observed for copulation for 5-10 min. After copulation, females were provided with honey and one to two hosts, depending on the experiment for parasitizing and then incubated under constant light at $25^{\circ} \mathrm{C}$.

\section{$F_{1}$ egg, larva, and pupa counts}

Each mated female was provided honey and one unparasitized fly host with only the anterior end of the fly pupa protruding from a foam plug. This restricted the females' ovipositing and thus localized egg laying to the anterior region of the fly pupae. Twenty-four hours later, the fly hosts were removed for offspring counts and replaced in the foam plug with new, unparasitized hosts. To characterize the $F_{1}$ hybrid incompatibility, we reciprocally crossed $N$. vitripennis (strain 13.2) and $N$. longicornis (strain IV7R3-1b, hereafter referred to as R3) to produce $F_{1}$ hybrids (Fig. 1b). Concurrently, we set up control self-crosses. All mating pairs are denoted as male $x$ female. For all crosses, females were hosted once with two hosts for $24 \mathrm{~h}$, and then these hosts were placed in the incubator. The Nasonia developed into pupae over 12-18 days before pupa counts were performed, Fig. 2a $(13.2 \times 13.2, n=8 ; \mathrm{R} 3 \times 13.2, n=3 ; 13.2 \times \mathrm{R} 3, n=5 ; \mathrm{R} 3$ $\times$ R3, $n=5$ ). For all counts, some offspring were not counted until they had already emerged from the pupal stage to adulthood. For the experimental crosses in Fig. $2 \mathrm{~b}$, the females were hosted twice, and one hosting was used for pupa counts $(13.2 \times 13.2, n=23 ; 12.1 \mathrm{~T} \times$ 12.1T, $n=13 ; 13.2 \times \mathrm{R} 3, n=23 ; 12.1 \mathrm{~T} \times \mathrm{R} 3, n=23 ; \mathrm{R} 3 \times$ $\mathrm{R} 3, n=25)$.
To validate the strong $F_{1}$ hybrid reduction and examine its dependency on strain background, we set up two interspecific crosses using $N$. longicornis R3 females and either $N$. vitripennis 13.2 or $12.1 \mathrm{~T}$ males that originated from the same inbred N. vitripennis strain in 1996 [23] (Fig. 2b). For the crosses in Fig. 2b after the hatch mark, the females were hosted six times with the fifth set used for pupa counts (Fig. 2b, $13.2 \times 13.2, n=5 ; 13.2 \times \mathrm{UT}, n$ $=5$; UT $\times \mathrm{UT}, n=14$ ).

The severe reduction of $F_{1}$ hybrids could be due to either a decrease in egg production or hybrid mortality during early developmental periods. The second set of hostings was used for egg counts to test for differences in fecundity (Fig. 2c, R3 $\times$ R3, $n=17 ; 13.2 \times \mathrm{R} 3, n=12$; $13.2 \times \mathrm{UT}, n=5$; UT $\times \mathrm{UT}, n=15)$. Egg counts entail carefully puncturing the fly's puparium, removing its anterior portion, and then counting all Nasonia eggs observed on the fly body and in the removed section of the puparium. The third of these hostings was used for first instar larva counts to test the nature of the reduction, (Fig. 2c, R3 $\times$ R3, $n=18$; $13.2 \times$ R3, $n=10 ; 13.2 \times$ $\mathrm{UT}, n=6$; UT $\times \mathrm{UT}, n=19$ ). To calculate the number of surviving first instar larvae, the same procedure for egg counts was used except that the Nasonia embryos were allowed to develop undisturbed on the host for one and a half days before being counted.

Next, we determined if the laboratory-evolved asymmetric hybrid mortality is due to a cytonuclear interaction or maternal genetic effect by generating reciprocal $F_{1}$ heterozygous females between $N$. longicornis strains R3 and the compatible UT strain, resulting in identical nuclear genomes but cytotypes derived from either R3 or UT (Fig. 1c). For the heterozygous experimental crosses, one half of the hosts for each cross were used for egg counts the day after hosting and the other half were incubated for another 12 days and used for pupa counts. The same procedure was used for the
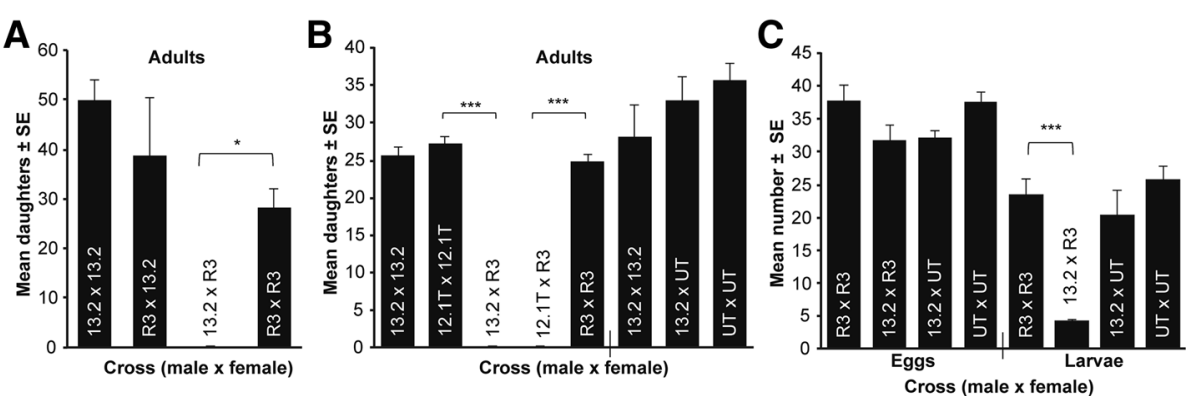

Fig. $2 F_{1}$ hybrid mortality is asymmetric and embryonic. a Complete $F_{1}$ hybrid reduction occurs between $N$. vitripennis males (strain 13.2$)$ and $N$. longicornis females (strain R3). Data are represented as mean number of surviving $F_{1}$ females (diploid) \pm standard error (SE), Kolmogorov-Smirnov $(\mathrm{K}-\mathrm{S})$ test, ${ }^{*} P<0.05$. b The factor responsible for complete $\mathrm{F}_{1}$ hybrid reduction in $N$. vitripennis is common to $N$. vitripennis strains 13.2 and $12.1 T$ that shared an ancestor in 1996. Conversely, the factor responsible for complete $F_{1}$ hybrid reduction in $N$. longicornis is specific to the Utahderived strain $\mathrm{R} 3$, but not UT. Data are $\mathrm{F}_{1}$ females $\pm \mathrm{SE}, \mathrm{K}-\mathrm{S}$ test, ${ }^{* * *} P<0.0001$, c Complete $\mathrm{F}_{1}$ hybrid reduction is due to hybrid mortality during embryonic development. Data are shown as mean number of $F_{1}$ eggs and $1^{\text {st }}$ instar larvae $\pm S E, K-S$ test, ${ }^{* * *} P<0.0001$ 
second hosting except the hosting group halves were switched. The hostings for pupae in Fig. 3a came after the hostings for pupae in Fig. 3b (Fig. 3a, UT $\times$ UT/R3, $n=14 ; 13.2 \times \mathrm{UT} / \mathrm{R} 3, n=14 ; 13.2 \times \mathrm{R} 3 / \mathrm{UT}, n=13 ; \mathrm{R} 3 \times$ R3/UT, $n=11$; Fig. 3b UT $\times$ UT/R3, $n=9 ; 13.2 \times \mathrm{UT} / \mathrm{R} 3$, $n=12 ; 13.2 \times \mathrm{R} 3 / \mathrm{UT}, n=10 ; \mathrm{R} 3 \times \mathrm{R} 3 / \mathrm{UT}, n=9$ ).

To corroborate the interpretation of a maternal effect hybrid incompatibility, we generated two $N$. longicornis introgression lines by backcrossing the R3 nuclear genotype into the UT cytotype (denoted IntR3/UT) and reciprocally the UT nuclear genotype into the R3 cytotype (denoted IntUT/R3) for nine generations each (Fig. 1d). These introgression lines harbor $\sim 99.8 \%$ of the nuclear genotype of one $N$. longicornis strain while maintaining the cytotype of the alternate strain. If the hybrid mortality is due to a maternal effect in hybrid embryos, then only the introgression line with the R3 nuclear DNA will result in hybrid death. For the introgression crosses, females were hosted six times with the fifth set used for pupa counts (Fig. 5a, IntUT/R3 $\times$ IntUT/R3, $n=16 ; 13.2 \times$ IntUT/R3, $n=12 ; 13.2 \times$ IntR3/ $\mathrm{UT}, n=7 ; \quad$ IntR3/UT $\times$ IntR3/UT, $n=17$ ). Using introgression-heterozygotes we can retest the observations of Fig. 4 but with introgressed genomes within the alternative $N$. longicornis cytotypes. For the introgressionheterozygote crosses, we generated reciprocal $F_{1}$ heterozygotes by backcrossing the introgression lines to males of their cytotype to generate R3/(IntUT/R3) and UT/(IntR3/UT) hybrids derived from the genotypes of father/mother (Fig. 5b).
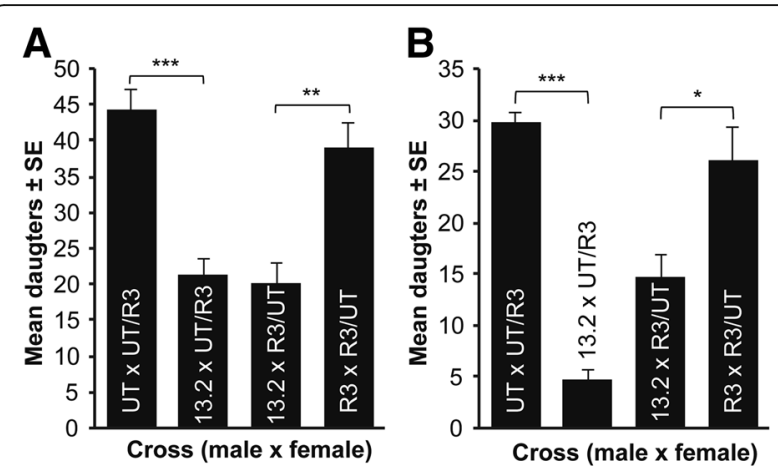

Fig. $4 F_{1}$ hybrid mortality is due to a maternal genetic effect. a and b $F_{1}$ hybrid reductions occur between $N$. vitripennis males (strain 13.2) and reciprocal N. longicornis herterozygous females (UT/R3 and R3/UT) for replicate experiments. Data are shown as mean number of $\mathrm{F}_{1}$ adult females $\pm \mathrm{SE}, \mathrm{K}-\mathrm{S}$ test, ${ }^{*} P<0.05$, ${ }^{* *} P<0.01$, and ${ }^{*}{ }^{*} P<$ 0.0001 . Crossing labels of R3/UT and UT/R3 denote heterozygous mothers derived from parents that were male/female

Females were hosted five times with the second set of hosts used for pupa counts as previously described (Fig. 5b, R3/(IntUT/R3) $\times$ R3/(IntUT/R3), $n=19 ; 13.2 \times$ R3/(IntUT/R3), $n=16 ; 13.2 \times \mathrm{UT} /(\mathrm{IntR} 3 / \mathrm{UT}), n=21$; $\mathrm{UT} /(\mathrm{IntR} 3 / \mathrm{UT}) \times \mathrm{UT} /(\mathrm{IntR} 3 / \mathrm{UT}), n=20)$.

\section{Statistical analysis}

Crosses that produced no offspring were excluded from the counts, likely due to poor fly host quality (average of

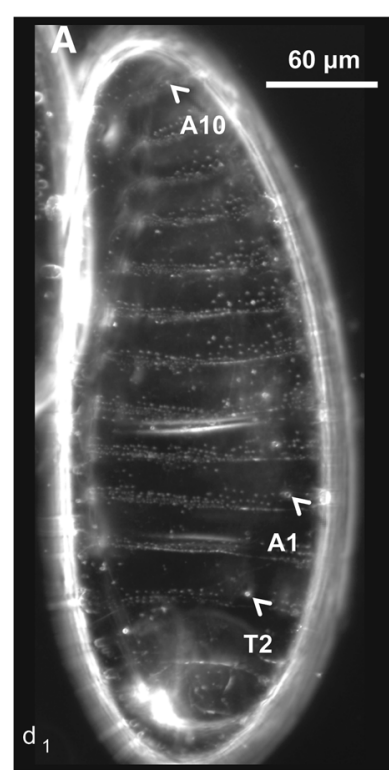

$\mathrm{R} 3 \times \mathrm{R} 3$

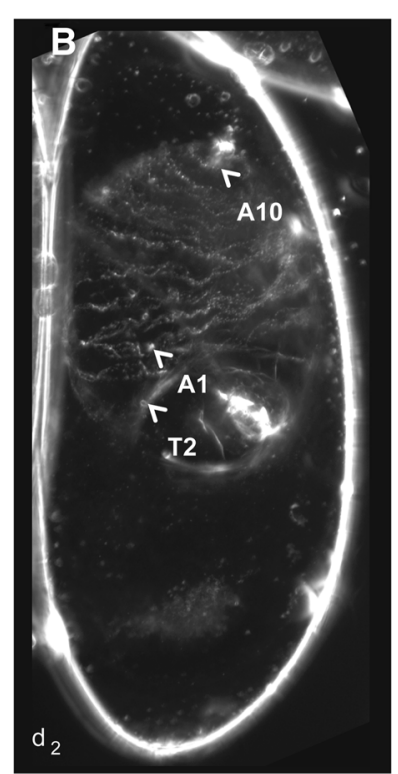

$13.2 \times \mathrm{R} 3$

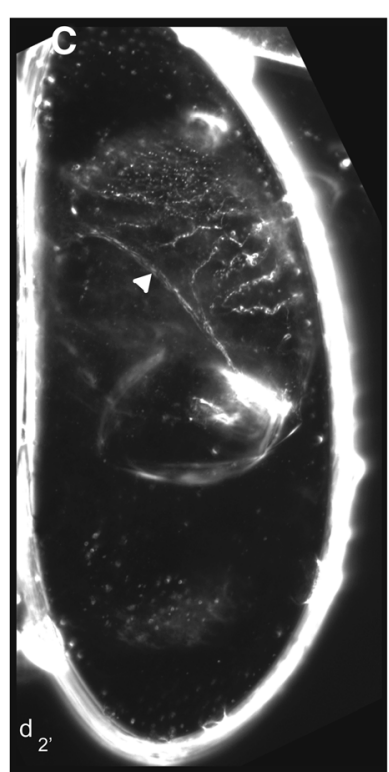

$13.2 \times \mathrm{R} 3$

Fig. 3 Dark field imagery of $36 \mathrm{~h}$ embryos. $\left(\mathbf{a}_{1} \mathrm{~d}_{1}\right) A n R 3 \times R 3$ larval cuticle just before hatching (pre-eclosure), $T 2$ arrow indicates $2^{\text {nd }}$ thoracic spiracle, A1 arrow indicates $1^{\text {st }}$ abdominal spiracle, A10 arrow indicates $10^{\text {th }}$ abdominal deticle belt. $\left(\mathbf{b}, d_{2}\right)$ a representative unhatched $13.2 \times R 3$ cuticle at two focal planes. $\left(\mathbf{c}, \mathrm{d}_{2^{\prime}}\right.$ ) An unhatched $13.2 \times$ R3 cuticle at two focal planes, closed arrow in indicates the edge of cuticle bordering the dorsal hole indicating a failure in dorsal closure 

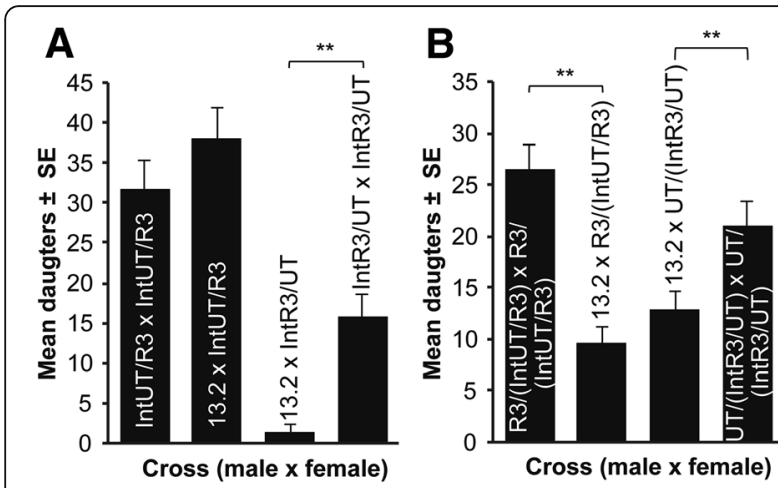

Fig. 5 Introgression experiments corroborate a maternal genetic effect on hybrid mortality. a Introgression crosses. Nearly complete $\mathrm{F}_{1}$ hybrid mortality requires the R3 nuclear DNA, as evident by introgression of the R3 genome into the UT cytoplasm. Data are shown as mean number of adult females \pm $\mathrm{SE}, \mathrm{K}-\mathrm{S}$ test, ${ }^{*} P<0.01$. b Introgression-heterozygote crosses. $\mathrm{F}_{1}$ hybrid mortality is partial when mothers are heterozygous for the incompatible R3 and compatible UT genotypes. Data are shown as mean number of females $\pm \mathrm{SE}, \mathrm{K}-\mathrm{S}$ test, ${ }^{*} P<0.01$

$4.9 \% \pm 2.1 \%)$. Crosses in which failure of fertilization occurred, as evident by all male offspring, were also excluded. Crosses that produced more than three offspring that were in diapause or were otherwise unidentifiable by sex were excluded from further analysis to avoid artifacts in the analysis because sex is unknown in the diapaused larvae. Kolmogorov-Smirnov (K-S) tests were used to compare statistical distributions of the number of daughters between each interspecific cross and corresponding intraspecific control cross for each experiment. The statistical package JMP 11 was used to perform Kolmogorov-Smirnov tests to compare egg production, female adult production, male adult production, and larva production between each interspecific cross and corresponding intraspecific control cross.

\section{Testing for Wolbachia infection}

To confirm the uninfected Wolbachia status of the Nasonia strains used, DNA was extracted using the Gentra PureGene DNA extraction kit (QIAGEN ${ }^{\circ}$ ), as well as a control infected strain (N. vitripennis 12.1). Verification of infection status was performed using PCR with the primers ftsZuniF and fts2uniR as previously described [25]. Individual adult Nasonia, $n=4 \mathrm{UT}$, $n=3$ R3, and $n=3$ 13.2, $n=3$ Wolbachia positive Nasonia - strain 12.1, were tested and no Wolbachia was detected in the UT, R3, or 13.2 strains.

\section{Cuticle prep methods}

To test embryonic and pre-eclosing larval development, eggs were collected from three R3 $\times$ R3 and three 13.2 $\times$ R3 mated females. Mated females laid eggs overnight at $25{ }^{\circ} \mathrm{C}$ in individual egg laying chambers. Eggs were collected to 1\% PBS/agarose plates and incubated at $25{ }^{\circ} \mathrm{C}$ for $\sim 48 \mathrm{~h}$. Unhatched eggs and empty cuticles were counted from each type of mating. Unhatched eggs were transferred to a drop of 90:10 lactic acid:ethanol on a glass slide, covered with $22 \times 22 \mathrm{~mm}$ cover glass and baked at $65{ }^{\circ} \mathrm{C}$ overnight. Some R3 $\times$ R3 eggs were collected and prepared after $\sim 30 \mathrm{~h}$ to catch them before hatching. Cleared cuticles were observed under dark field optics. Contrast and brightness were enhanced using Adobe Photoshop'.

\section{Results}

All mating pairs are denoted as male $\times$ female. Figure $2 \mathrm{a}$ shows that while there are no significant differences in hybrids produced in the interspecific cross $\mathrm{R} 3 \times 13.2$ and control cross $13.2 \times 13.2$ (Kolmogorov-Smirnov, $P=$ 0.97 , hereafter referred to as $\mathrm{K}-\mathrm{S})$, there is a significant and marked lack of hybrids observed in the reciprocal interspecific cross $13.2 \times \mathrm{R} 3$ compared to control cross R3 $\times$ R3 (K-S, $P=0.014)$. Thus, $F_{1}$ hybrid reduction is contingent on having a 13.2 father and R3 mother. Due to the haplodiploid sex determination of Nasonia, $\mathrm{F}_{1}$ haploid sons are not hybrids, develop from unfertilized eggs, and arise from the maternal genotype. Accordingly, in all of the inter- and intraspecific crosses above and hereafter, there are no significant differences in $F_{1}$ male survival (Fig. 2a, $13.2 \times \mathrm{R} 3$ compared to R3 $\times$ R3, K-S, $P$ $=0.769 ; 13.2 \times \mathrm{R} 3$ and $12.1 \mathrm{~T} \times \mathrm{R} 3$ compared to $\mathrm{R} 3 \times$ R3, K-S, $P=0.969$ and $P=0.405$ respectfully).

For the two interspecific crosses using $N$. longicornis R3 females and either $N$. vitripennis 13.2 or $12.1 \mathrm{~T}$ (Fig. 2b) males, there were no hybrids produced. Conversely, the R3 $\times$ R3 control cross yielded normal numbers of offspring (Fig. 2b, K-S, $P<0.0001$ for both comparisons). Since the underlying genetic factor for the hybrid reduction is presumably in both the $N$. vitripennis 13.2 and $12.1 \mathrm{~T}$ strains, it is likely that the incompatibility originated prior to their 1996 splitting [23]. Next, we assessed whether the incompatibility in $N$. longicornis is strain dependent by crossing 13.2 males with females from a second $N$. longicornis strain, NAS_NLUT 230A (hereafter referred to as UT). The UT and R3 strains were collected in Utah several decades ago. Figure $2 \mathrm{~b}$ shows that there was no reduction in $\mathrm{F}_{1}$ hybrids in the $13.2 \times$ UT cross compared to the control UT $\times$ UT cross (K-S, $P=0.6896)$. Therefore, the $F_{1}$ hybrid reduction is specific to the extant strain R3 that was previously compatible with the same $N$. vitripennis strain 13.2 in the year 2000 [16]. Likewise, when R3 was reestablished from larval diapause stocks from 2008, we again observed $\mathrm{F}_{1}$ hybrid reduction in the $13.2 \times \mathrm{R} 3$ interspecific cross compared to that of the self R3 $\times$ R3 (K-S, $P=$ 0.0135). Thus, we calibrate the spread of the $N$. 
longicornis R3 incompatibility factor to an eight-year time span between the years 2000 and 2008 .

For egg counts, we observed no significant differences in fecundity between the interspecific cross $13.2 \times \mathrm{R} 3$ and the control cross R3 $\times$ R3 (Fig. 2c, K-S, $P=0.22$ ). Thus, the decrease in $F_{1}$ hybrids is due to postzygotic hybrid mortality. We observed a significant $82.4 \%$ reduction in the first instar larvae in the $13.2 \times \mathrm{R} 3$ cross relative to the R3 $\times$ R3 control (Fig. 2c, K-S, $P<0.0001$ ), indicating the hybrid mortality is primarily embryonic; the surviving larvae are haploid males. Embryonic mortality is also evident by microscopy in which $12 / 65$ progeny from the R3 $\times \mathrm{R} 3$ crosses failed to hatch after more than $36 \mathrm{~h}$, while 66/70 from the $13.2 \times \mathrm{R} 3$ hybrid eggs failed to hatch (Chi-squared test, $P<0.0001$ ). The hatched embryos were haploid males and not hybrids. Of the unhatched hybrid embryos, many were tiny, malformed, and clearly inviable. The cuticles of the inviable larvae had all three thoracic and ten abdominal segments, but were highly compressed (Fig. 3). Many also showed large openings on the dorsal side of the cuticle, indicating a failure in dorsal closure. Similar phenotypes have been observed in screens for embryonic lethal mutations in Nasonia [26] and Drosophila [27]. These results imply that hybrid lethality likely affects a specific developmental process, such as dorsal-ventral patterning or extraembryonic membrane specification.

The $N$. longicornis heterozygous genotypes are denoted $\mathrm{UT} / \mathrm{R} 3$ and R3/UT and represent paternal/maternal origins. We crossed these heterozygous females with $N$. vitripennis 13.2 males in two replicate experiments, and observed significant hybrid reductions in crosses to both R3/UT or UT/R3 females (Fig. 4a and b). These results exclude the R3 cytotype causing hybrid mortality, and are consistent with a maternal genetic effect incompatibility between the maternal R3 genotype and 13.2 embryonic genotype, irrespective of the $N$. longicornis cytotype.

The $N$. longicornis introgression lines used to test a maternal effect on hybrid incompatibility, IntR3/UT and IntUT/R3, exhibited an asymmetric F1 hybrid lethality when crossed to $N$. vitripennis 13.2 males (Fig. 5a). Specifically, $92 \%$ hybrid mortality occurred in the incompatible cross with $N$. longicornis females containing a majority R3 genome and a UT cytoplasm (IntR3/UT). In contrast, $N$. longicornis females with the UT genome and R3 cytoplasm produced slightly more offspring (hybrids) than the control self-cross. These and the aforementioned results validate the contingency of the hybrid mortality on a R3 nuclear genotype expressed maternally during oogenesis, that in turn negatively interacts with $N$. vitripennis nuclear genes in the embryo.

We found no hybrid lethality in the crosses with heterozygous or introgression lines between the two $N$. longicornis strains, indicating that the incompatibility arose specifically between R3 and $N$. vitripennis. It is important to note that although the control IntR3/UT cross in Fig. 5a is not as fecund as the IntUT/R3 line, giving the appearance of a potential cytonuclear incompatibility between R3 genotype and UT cytotype, there is no significant difference between egg and adult production for this line (K-S, $P=0.675)$. Therefore, the incompatibility exhibited between species is not observed between strains withing the $N$. longicornis species.

In Fig. $5 \mathrm{~b}$, we generated reciprocal $\mathrm{F}_{1}$ heterozygotes using the introgression lines, R3/(IntUT/R3) and UT/ (IntR3/UT); these heterozygous genotypes contain different $N$. longicornis cytotypes, but the same $N$. longicornis heterozygous nuclear genotypes. If hybrid mortality with 13.2 males is due to the proposed interaction between the R3 maternal genotype and $N$. vitripennis 13.2 embryonic genotype, then $N$. longicornis heterozygous mothers between R3 and UT should again yield partial mortality. Indeed, Fig. 5b shows that there was approximately a $50 \%$ reduction of daughters produced in both cross directions, as expected.

\section{Discussion}

The experiments presented here demonstrate, for the first time, that complete $F_{1}$ hybrid lethality evolved in the laboratory between $N$. vitripennis males and $N$. longicornis females between 2000 [20] to 2008. Assuming a two-week generation time, the incompatibility spread in less than 209 generations in cultures typically maintained with less than thirty foundresses per generation. The $\mathrm{F}_{1}$ hybrid mortality is in part due to a $N$. longicornis R3 maternal genetic effect, rather than the R3 cytotype. This conclusion is principally based on experiments crossing $N$. vitripennis males to reciprocal $F_{1}$ heterozygous females between $N$. longicornis strains $\mathrm{R} 3$ and the compatible UT strain that vary in cytotype. If hybrid mortality was due to a cytonuclear interaction - which is common in $F_{2}$ hybrid males of Nasonia [18-20] - then hybrid mortality would have been unidirectional and contingent on parental females harboring the R3 cytotype. Instead, we observed hybrid mortality in both crosses. Moreover, there mortality appears to involve an interaction between an embryonic product of the $N$. vitripennis genome and a maternal product of the R3 genome that is partially dominant or codominant with the maternal UT allele(s), since approximately half of the progeny of the heterozygous $N$. longicornis (UT/R3 or R3/UT) females succumb to lethality. Given that the embryo lethality seems to be related to a fairly narrow set of developmental processes (the tightly intertwined dorsoventral (DV) patterning and extraembryonic membrane specification processes) [28], a single locus developmental gene (e.g., transcription factor or signal 
transduction pathway component) would be a plausible genetic part of the hybrid incompatibility, though this inference awaits future experimentation.

It is possible that a polymorphism within the species of $N$. longicornis ultimately spread in the R3 line via correlated selection or drift to cause the $F_{1}$ hybrid incompatibility, as reviewed in [29]. While selection or drift may have fixed the polymorphism from standing genetic variation in the species, the relatively few foundresses that established the R3 line and the subsequent inbreeding and bottlenecking of laboratory stocks likely reduced genetic variability and had an influence on the relatively sudden fixation within the laboratory. Previous laboratory experiments on the evolution of reproductive isolation found that selection can drive fixation of traits and reproductive barriers in a short period of time (as reviewed in [30, 31]). Experimental evolution of Drosophila melanogaster on a diet of EDTA led to a high degree of variability in fitness/fecundity in $\mathrm{F}_{1}$ hybrid crosses between the control and adapted strains [32]. Following these observations, several generations of introgression switched the third chromosome of a control strain into the background of the EDTAadapted strain, resulting in complete sterility of females on all diets as well as lethality of both sexes at highEDTA conditions.

While the sudden appearance of genetically-based hybrid embryonic lethality in animal hybrids is very rare, embryonic lethality in hybrid embryos occurs in other insects. For instance, several interspecific crosses within the Drosophila genus lead to embryonic lethality [33-35]. Similar to the incompatibility in Nasonia, these defects are often sex specific and depend on the direction of the cross. For example, $D$. melanogaster males $\times D$. simulans females produce lethality in female embryos [34], D. melanogaster females $\times D$. santomea males produce dead male embryos [35], and D. montana females $\times D$. texana males leads to complete female embryo lethality in early development [33]. Unlike Nasonia, which lack sex chromosomes, the sex specificity in Drosophila seems to arise from negative interactions between the X-chromosome of one species and autosomal loci of the other. In addition, the Drosophila species pairs diverged 3-11 million years ago while $N$. longicornis and $N$. vitripennis diverged approximately one million years ago.

Despite these major differences between insect systems, some observations in Drosophila are relevant for the rapid appearance of hybrid embryonic lethality observed in Nasonia. A survey of $D$. simulans and $D$. santomea populations found that there is natural variation affecting the strength or presence of the hybrid embryonic lethality when crossed to D. melanogaster $[35,36]$ These and other cases in Arabidopsis [11] and Tribolium [12, 37], suggest that alleles affecting hybrid incompatibilities are segregating within populations. However, it is unclear if within-species genetic variation for hybrid incompatibilities spread recently or in the distant past. The rate at which lineages evolve intrinsic postzygotic isolation has been benchmarked against the rate of prezygotic isolation evolution [38-41]. For example, strains of allopatric Drosophila can have equal rates of divergence for pre- and post-zygotic reproductive isolation, while birds develop postzygotic barriers much slower [38, 41, 42]. Extremely small population sizes and relatively short time frames have also never been linked to the evolution of hybrid mortality [31]. This lack of knowledge on the tempo of evolutionary genetic changes affecting hybrid incompatibilities suggests that the spread of alleles underlying geneticallybased hybrid mortality may be unusually prolonged in time and/or restricted to large populations.

In contrast, there is some evidence for the rapid evolution of bottleneck-induced premating isolation $[1,4,43]$. Various speciation theories propose that evolution (i.e., selection and/or genetic drift) in small demes can cause the spread of a particular set of traits and genetic makeup, which may lead to strong reproductive isolation [44]. For instance, hybrid lethality between species could suddenly arise if selection or population bottlenecks fix segregating variation for hybrid lethality. In the present work, we observed the rapid evolution of a complete, asymmetric, $F_{1}$ hybrid mortality within eight years of routine laboratory rearing. These laboratory populations are maintained as small, inbred populations where strong selection or genetic drift may have rapidly promoted the spread of allele(s) for complete $F_{1}$ interspecific hybrid lethality.

\section{Conclusions}

Here we have characterized the establishment of severe F1 hybrid mortality in observable time. The asymmetric incompatibility spread in less than eight years under laboratory maintenance of small populations of a strain of $\mathrm{N}$. longicornis and is likely due to a negative interaction between a strain-specific maternal genetic effect and a nuclear-encoded product derived from $\mathrm{N}$. vitripennis in F1 hybrid embryos. Hybrid mortality appears related to a disruption of early embryonic patterning, where maternal effects and the zygotic genome first interact.

\section{Abbreviations}

Int: Introgression; K-S: Kolmogorov-Smirnov; R3: IV7R3-1b; UT: NAS_NLUT230A

\section{Acknowledgements}

We would like to thank Gina Qin for her help in preliminary work on the projects. This research was made possible by NSF award DEB 1046149 and IOS 1456778 to S.R.B. Support for R.M.B was made possible by the Rowland Institute at Harvard University.

Availability of data and materials

All stock lines are maintained at Vanderbilt University and University of Chicago and available upon request. 


\section{Authors' contributions}

AS contributed to the design and execution of the experimental matings and embryogenesis observations as well as writing the manuscript. MP contributed to the execution of experimental matings. AN contributed by conducting the diapause line experimental matings. JAL contributed by conducting the embryogenesis work and writing the manuscript, SRB contributed to the design of the experiments and the writing of the manuscript. RMB contributed to the design and execution of the experimental mating crosses and embryogenesis observations as well as writing the manuscript. All authors read and approved the final manuscript.

\section{Competing interests}

The authors declare that they have no competing interests.

\section{Consent for publication}

Not applicable

\section{Ethics approval}

Not applicable.

\section{Author details}

'Department of Biological Sciences, Vanderbilt University, Nashville, TN 37235, USA. ${ }^{2}$ Present Address: Department of Biology, University of Utah, Salt Lake City, UT 84112, USA. ${ }^{3}$ Present Address: Children's Hospital Los Angeles, Los Angeles, CA 90027, USA. ${ }^{4}$ Department of Biological Sciences, University of Illinois at Chicago, Chicago, USA. ${ }^{5}$ Department of Pathology, Microbiology, and Immunology, Vanderbilt University, Nashville, TN 37235, USA. ${ }^{6}$ The Rowland Institute at Harvard University, Harvard University, 100 Edwin H. Land Blvd, Cambridge, MA 02142, USA

\section{Received: 25 September 2016 Accepted: 10 January 2017} Published online: 26 January 2017

\section{References}

1. Crossley SA. Changes in mating-behavior produced by selection for ethological isolation between ebony and vestigial mutants of drosophila-melanogaster. Evolution. 1974;28(4):631-47.

2. del Solar E. Sexual isolation caused by selection for positive and negative phototaxis and geotaxis in Drosophila pseudoobscura. Proc Natl Acad Sci U S A. 1966:56(2):484-7.

3. Kilias G, Alahiotis SN, Pelecanos M. A multifactorial genetic investigation of speciation theory using Drosophila melanogaster. Evolution. 1980;34(4):730-7.

4. Dodd DMB. Reproductive isolation as a consequence of adaptive divergence in Drosophila pseudoobscura. Evolution. 1989;43(6):1308-11.

5. Dettman JR, Sirjusingh C, Kohn LM, Anderson JB. Incipient speciation by divergent adaptation and antagonistic epistasis in yeast. Nature. 2007:447(7144):585-8.

6. Curnoe D, Thorne A, Coate JA. Timing and tempo of primate speciation. J Evolution Biol. 2006;19(1):59-65.

7. Fitzpatrick BM. Rates of evolution of hybrid inviability in birds and mammals. Evolution. 2004;58(8):1865-70

8. Price TD, Bouvier MM. The evolution of F1 postzygotic incompatibilities in birds. Evolution. 2002;56(10):2083-9.

9. Sasa MM, Chippindale PT, Johnson NA. Patterns of postzygotic isolation in frogs. Evolution. 1998;52(6):1811-20.

10. Lijtmaer DA, Mahler B, Tubaro PL. Hybridization and postzygotic isolation patterns in pigeons and doves. Evolution. 2003;57(6):1411-8.

11. Bomblies K, Weigel D. Hybrid necrosis: autoimmunity as a potential gene-flow barrier in plant species. Nat Rev Genet. 2007;8(5):382-93.

12. Wade MJ, Johnson NA. Reproductive isolation between two species of flour beetles, Tribolium castaneum and T. freemani: variation within and among geographical populations of T. castaneum. Heredity. 1994;72:155-62.

13. Raychoudhury R, Desjardins CA, Buellesbach J, Loehlin DW, Grillenberger BK, Beukeboom L, Schmitt T, Werren JH. Behavioral and genetic characteristics of a new species of Nasonia. Heredity. 2010;104(3):278-88.

14. Dittmer J, van Opstal EJ, Shropshire JD, Bordenstein SR, Hurst GDD, Brucker RM. Disentangling a Holobiont - recent advances and perspectives in Nasonia wasps. Front Microbiol. 2016;7:1478

15. Bordenstein SR, O'Hara FP, Werren JH. Wolbachia-induced incompatibility precedes other hybrid incompatibilities in Nasonia. Nature. 2001;409(6821):707-10.
16. Bordenstein SR, Werren JH. Bidirectional incompatibility among divergent Wolbachia and incompatibility level differences among closely related Wolbachia in Nasonia. Heredity. 2007;99(3):278-87.

17. Beukeboom LW, van den Assem J. Courtship and mating behaviour of interspecific Nasonia hybrids (Hymenoptera, Pteromalidae): A grandfather effect. Behav Genet. 2001;31(2):167-77.

18. Breeuwer JAJ, Werren JH. Hybrid breakdown between two haplodiploid species: the role of nuclear and cytoplasmic genes. Evolution. 1995;49(4):705-17.

19. Koevoets T, Niehuis O, van de Zande L, Beukeboom LW. Hybrid incompatibilities in the parasitic wasp genus Nasonia: negative effects of hemizygosity and the identification of transmission ratio distortion loci. Heredity. 2012;108(3):302-11.

20. Niehuis O, Judson AK, Gadau J. Cytonuclear genic incompatibilities cause increased mortality in male F2 hybrids of Nasonia giraulti and $N$. vitripennis. Genetics. 2008:178(1):413-26.

21. Bordenstein SR, Drapeau MD, Werren JH. Intraspecific variation in sexual isolation in the jewel wasp Nasonia. Evolution. 2000:54(2):567-73.

22. Brucker RM, Bordenstein SR. The hologenomic basis of speciation: gut bacteria cause hybrid lethality in the genus Nasonia. Science. 341(6146):667-69.

23. Perrot-Minnot MJ, Guo LR, Werren JH. Single and double infections with Wolbachia in the parasitic wasp Nasonia vitripennis: Effects on compatibility. Genetics. 1996;143(2):961-72

24. Chafee ME, Zecher CN, Gourley ML, Schmidt VT, Chen JH, Bordenstein SR, Clark ME, Bordenstein SR. Decoupling of host-symbiont-phage coadaptations following transfer between insect species. Genetics. 2011;187(1):203-15.

25. Baldo L, Dunning Hotopp JC, Jolley KA, Bordenstein SR, Biber SA, Choudhury RR, Hayashi C, Maiden MC, Tettelin H, Werren JH. Multilocus sequence typing system for the endosymbiont Wolbachia pipientis. Appl Environ Microbiol. 2006;72(11):7098-110.

26. Pultz MA, Zimmerman KK, Alto NM, Kaeberlein M, Lange SK, Pitt JN, Reeves $\mathrm{NL}$, Zehrung DL. A genetic screen for zygotic embryonic lethal mutations affecting cuticular morphology in the wasp Nasonia vitripennis. Genetics. 2000;154(3):1213-29.

27. Jurgens G, Wieschaus E, Nussleinvolhard C, Kluding H. Mutations affecting the pattern of the larval cuticle in Drosophila melanogaster. II. Zygotic loci on the third chromosome. Roux Arch Dev Biol. 1984;193(5):283-95.

28. Ozuak O, Buchta T, Roth S, Lynch JA. Dorsoventral polarity of the Nasonia embryo primarily relies on a BMP gradient formed without input from toll. Curr Biol. 2014;24(20):2393-8

29. Cutter AD. The polymorphic prelude to Bateson-Dobzhansky-Muller incompatibilities. Trends Ecol Evol. 2012;27(4):209-18.

30. Rice WR, Hostert EE. Laboratory experiments on speciation: what have we learned in 40 years? Evolution. 1993:47(6):1637-53.

31. Odeen A, Florin AB. Effective population size may limit the power of laboratory experiments to demonstrate sympatric and parapatric speciation. Proc Biol Sci. 2000;267(1443):601-6.

32. Robertson FW. The ecological genetics of growth in Drosophila. 8. Adaptation to a new diet. Genet Res. 1966;8(2):165-79.

33. Kinsey JD. Studies on an embryonic lethal hybrid in Drosophila. Development. 1967;17(2):405-23.

34. Hadorn E. Zur Autonomie und Phasenspezifität der Letalität von Bastarden zwischen Drosophila melanogaster und Drosophila simulans. Genève: Kundig; 1961.

35. Gavin-Smyth J, Matute DR. Embryonic lethality leads to hybrid male inviability in hybrids between Drosophila melanogaster and D. santomea. Ecol Evol. 2013;3(6):1580-9.

36. Gerard PR, Presgraves DC. Abundant genetic variability in Drosophila simulans for hybrid female lethality in interspecific crosses to Drosophila melanogaster. Genet Res. 2012;94(1):1-7.

37. Wade MJ, Johnson NA, Jones R, Siguel V, McNaughton M. Genetic variation segregating in natural populations of Tribolium castaneum affecting traits observed in hybrids with T. freemani. Genetics. 1997;147(3):1235-47.

38. Coyne JA, Orr HA. "Patterns of speciation in Drosophila" Revisited. Evolution. 1997;51(1):295-303.

39. Kozak GM, Rudolph AB, Colon BL, Fuller RC. Postzygotic isolation evolves before prezygotic isolation between fresh and saltwater populations of the rainwater killifish, Lucania parva. Int J Evol Biol. 2012;2012:523967.

40. Yukilevich R. Asymmetrical patterns of speciation uniquely support reinforcement in Drosophila. Evolution. 2012:66(5):1430-46.

41. Rabosky DL, Matute DR. Macroevolutionary speciation rates are decoupled from the evolution of intrinsic reproductive isolation in Drosophila and birds. Proc Natl Acad Sci U S A. 2013;110(38):15354-9. 
42. Orr HA, Coyne JA. The genetics of postzygotic isolation in the Drosophila virilis group. Genetics. 1989;121(3):527-37.

43. Matute DR. The role of founder effects on the evolution of reproductive isolation. J Evolution Biol. 2013;26(11):2299-311.

44. Templeton AR. The theory of speciation via the founder principle. Genetics. 1980;94(4):1011-38.

Submit your next manuscript to BioMed Central and we will help you at every step:

- We accept pre-submission inquiries

- Our selector tool helps you to find the most relevant journal

- We provide round the clock customer support

- Convenient online submission

- Thorough peer review

- Inclusion in PubMed and all major indexing services

- Maximum visibility for your research

Submit your manuscript at www.biomedcentral.com/submit 